\title{
Sulfonylurea in combination with insulin is associated with increased mortality compared with a combination of insulin and metformin in a retrospective Danish nationwide study
}

\author{
Ulrik M. Mogensen • Charlotte Andersson • Emil L. Fosbøl • Tina K. Schramm • \\ Allan Vaag • Nikolai M. Scheller • Christian Torp-Pedersen • Gunnar Gislason • \\ Lars Køber
}

Received: 6 April 2014 / Accepted: 14 August 2014 /Published online: 10 September 2014

(C) Springer-Verlag Berlin Heidelberg 2014

\begin{abstract}
Aims/hypothesis Individual sulfonylureas (SUs) and metformin have, in some studies, been associated with unequal hypoglycaemic, cardiovascular and mortality risks when used as monotherapy in type 2 diabetes. We investigated the outcomes in patients treated with different combinations of SUs
\end{abstract}

Electronic supplementary material The online version of this article (doi:10.1007/s00125-014-3372-z) contains peer-reviewed but unedited supplementary material, which is available to authorised users.

U. M. Mogensen $(\bowtie) \cdot$ E. L. Fosbøl $\cdot$ L. Køber

The Heart Centre, Department of Cardiology, University Hospital

Rigshospitalet, 9441, Blegdamsvej 9, 2100 Copenhagen, Denmark e-mail: ulrik_mogensen@hotmail.com

C. Andersson · G. Gislason

Department of Cardiology, University Hospital Gentofte,

Copenhagen, Denmark

T. K. Schramm

Department of Cardiology, University Hospital Frederiksberg,

Copenhagen, Denmark

A. Vaag

Department of Endocrinology, University Hospital Rigshospitalet,

Copenhagen, Denmark

N. M. Scheller $\cdot$ C. Torp-Pedersen

Department of Health Science and Technology, Aalborg University, Aalborg, Denmark

G. Gislason

National Institute of Public Health, University of Southern Denmark, Copenhagen, Denmark

G. Gislason

Faculty of Health and Medical Sciences, University of Copenhagen,

Copenhagen, Denmark and insulin vs a combination of metformin and insulin in a retrospective nationwide study.

Methods All Danish individuals using dual therapy with SU+ insulin or metformin + insulin without prior myocardial infarction (MI) or stroke were followed from 1 January 1997 to 31 December 2009 in nationwide registries. Risks of all-cause mortality, cardiovascular death, hypoglycaemia and a composite endpoint of MI, stroke and cardiovascular death were compared. Rate ratios (RR) $[95 \%$ CIs] were calculated using time-dependent multivariable Poisson regression analysis.

Results A total of 11,081 patients used SU+insulin and 16,910 used metformin+insulin. Patients receiving metformin + insulin were younger and had less comorbidity and a longer history of glucose-lowering treatment. SU+insulin was associated with higher mortality rates compared with metformin+insulin (76-126 vs 23 per 1,000 person-years). In adjusted analyses, $\mathrm{SU}+$ insulin was associated with increased allcause mortality (RR $1.81[1.63,2.01]$ ), cardiovascular death (RR $1.35[1.14,1.60])$ and the composite endpoint (RR 1.25 $[1.09,1.42])$ compared with metformin +insulin. Hypoglycaemia was more frequent with $\mathrm{SU}+$ insulin than with metformin+insulin (17-23 vs six events per 1,000 person-years) and was associated with increased mortality (RR 2.13 [1.97, 2.37]). There were no significant differences in risk between individual SUs in combination with insulin. Conclusions/interpretation In combination with insulin, the use of SUs was associated with increased mortality compared with metformin. There were no significant risk differences between SUs.

Keywords Cardiovascular disease $\cdot$ Mortality $\cdot$ Risk . Treatment · Type 2 diabetes mellitus 


\begin{tabular}{|c|c|}
\hline \multicolumn{2}{|c|}{ Abbreviations } \\
\hline ACCORD & $\begin{array}{l}\text { Action to Control Cardiovascular Risk in } \\
\text { Diabetes }\end{array}$ \\
\hline ADVANCE & $\begin{array}{l}\text { Action in Diabetes and Vascular Disease: } \\
\text { Preterax and Diamicron MR Controlled } \\
\text { Evaluation }\end{array}$ \\
\hline ATC & Anatomical Therapeutic Classification \\
\hline GLT & Glucose-lowering therapy \\
\hline IQR & Interquartile range \\
\hline MI & Myocardial infarction \\
\hline $\mathrm{RR}$ & Rate ratio \\
\hline SU & Sulfonylurea \\
\hline SUR & Sulfonylurea receptor \\
\hline
\end{tabular}

\section{Introduction}

The majority of patients with type 2 diabetes mellitus need more than one glucose-lowering drug to maintain long-term glycaemic control [1], but questions remain over the safety of certain combinations and to what extent they might be associated with increased mortality or cardiovascular risks. Metformin is considered to be the first-line glucose-lowering therapy (GLT) in type 2 diabetes and is recommended at all stages of the disease if tolerated $[2,3]$. A combination of insulin and a sulfonylurea (SU) was among the first widely used combination regimens [4]. This combination was found to improve glycaemic control in some studies [5-7] while others did not consider it clinically useful in most patients [8], and the combination is not recommended in clinical guidelines [2, 4]. It has in fact been speculated that the combination of insulin and an SU could be associated with increased mortality mediated through hypoglycaemia [9].

Experimental and clinical observational studies have suggested differences in risk associated with individual SUs [10-12]. Thus, glibenclamide (known as glyburide in the USA and Canada) has been associated with increased mortality compared with gliclazide $[10,11]$ and glimepiride [10]. Furthermore, the risk of hypoglycaemia has been reported to be higher with the use of glibenclamide compared with other SUs [12]. Previous studies have not been able to determine whether differences exist between combinations of SUs and insulin in terms of efficacy or safety [8], but it has been speculated that differential effects of SUs may underpin the differences in outcome seen in recent clinical trials [11, 13-15].

Using nationwide administrative registries in Denmark, we aimed to elucidate the contemporary use of SU and insulin in combination, and to compare the cardiovascular and mortality risks associated with their use against that of insulin used in combination with metformin in patients with type 2 diabetes mellitus.

\section{Methods}

Data sources In Denmark, all residents are provided with a unique personal registration number enabling a linkage at an individual level between nationwide administrative registries. All prescriptions dispensed from Danish pharmacies since 1995 are recorded in the Danish Registry of Medicinal Product Statistics and coded by the Anatomical Therapeutic Classification (ATC) system. The data include the amount and strength of the drug and the date of dispensing. Records on all hospital admissions in Denmark since 1978 are available in the Danish National Patient registry and include primary and secondary diagnoses, coded according to the ICD-10 (www. who.int/classifications/icd/en/; ICD-8 before 1994). Vital status for all Danish residents was obtained from the Danish National Population registry, where information on all deaths is registered within 2 weeks after their occurrence. Causes of death based on death certificates according to the ICD-10 were obtained from the Danish National Cause of Death Register.

Population and exposure to GLTS We included all individuals $\geq 18$ years who had not had a prior myocardial infarction (MI) or stroke and who used GLT with a dual combination of insulin and an SU or metformin after 1 January 1997. Treatment intervals were calculated for all types of glucoselowering drug including insulin (ATC code: A10A), metformin (A10B) and SUs: glibenclamide (A10BB01), glimepiride (A10BB12), tolbutamide (A10BB03), gliclazide (A10BB09) and glipizide (A10BB07). A continuous treatment period was assumed from the amount of the dispensed glucose-lowering drugs and the interval between up to four consecutively claimed prescriptions, if this was compatible with at least the minimal dose of the respective glucose-lowering drug [16]. All intervals were extended by 30 days to avoid artificial breaks in the calculated treatment durations. Sensitivity analyses without this extension were performed. Patients were included in the study at the time of the first claimed prescription of dual combination therapy with insulin and an SU or metformin.

Comorbidity, cardiovascular therapy and income Charlson Comorbidity Index scores were calculated for each patient based on discharge diagnoses up to 10 years prior to baseline [17]. Information on concomitant cardiovascular therapy included the use of statins (ATC code: C10AA), beta-blockers (C07), angiotensin-converting enzyme inhibitors/angiotensin receptor blockers (C09), calcium channel blockers (C08), other antihypertensive drugs (C02), aspirin (B01AC06), nitrates (C01D), vitamin $\mathrm{K}$ antagonists (B01AA), spironolactone (C03D) and digoxin (C01AA). The gross income 5 years prior to baseline was graded in quintiles as a measure of socioeconomic status. Furthermore, we included 
information on hypoglycaemic hospitalisations (ICD-10: E110E, E159, E160, E161 and E162).

Outcomes Measures of outcome comprised mortality, cardiovascular death and a composite endpoint of MI (ICD10: I21-I22), stroke (I61-I64) and cardiovascular death (I00-I99). To estimate the hypoglycaemic risk associated with insulin combination therapies after baseline, we used a first-time hypoglycaemic hospitalisation as an outcome measure in a multivariable analysis.

Statistics Time-dependent multivariable Poisson regression analysis was used to compare rate ratios (RRs) for all endpoints. In an analysis of the combined endpoint, the follow-up was censored after the occurrence of a first event of interest (MI, stroke or cardiovascular death). Follow-up started at baseline (the date of the first claimed prescription of insulin and SU or metformin) and ended when reaching a relevant endpoint or at 31 December 2009. Patients exposed to a relevant combination therapy contributed with time at risk to this particular combination until stopping or switching therapy, or reaching the end of the study. To account for changes in GLT during follow-up, the follow-up time was split at every change in glucose-lowering treatment, and the exposure status was modified accordingly for each date of change in therapy.

The exposure groups were metformin + insulin, $\mathrm{SU}+$ insulin or 'other therapy', which encompassed monotherapy, multitherapy ( $\geq 3$ drugs) and various other two-drug combinations.

All models were adjusted for age, sex, duration of any GLT use (in years), calendar year of inclusion, Charlson score, income and concomitant cardiovascular pharmacotherapy. To account for the use of prior monotherapy, a variable indicating the initial use of SU, metformin or insulin prior to the initiation of combination therapy was included in the model.

As an example, a patient initiating metformin treatment, switching to SU (due to impaired kidney function) and then additionally prescribed insulin (due to inadequate glucose control on SU) is included in the study at the date of adding insulin on top of SU. The duration of treatment for diabetes included in the model is the time in years since initiating the first GLT (metformin). A variable indicating initial monotherapy is 1 for metformin and is included in the model. Renal disease is included in the model as two points added to the Charlson score. The patient is registered as having used both SU and metformin prior to inclusion in the study and is therefore not included in the sensitivity analyses on patients who have used solely SU or metformin (in the attempts to eliminate the potential effects of prior variability in monotherapy use).

Metformin +insulin was used as a reference in the primary analyses. As a mean of comparing metformin +insulin with the overall use of SU+insulin, we performed additional analyses in which all combinations of $\mathrm{SU}+$ insulin were pooled. When comparing individual SUs, glimepiride+ insulin, involving the most widely used SU, was used as the reference.

The assumptions of the Poisson model were tested and found valid.

In a propensity score matched analysis, patients using metformin +insulin were matched (1:1) with patients using $\mathrm{SU}+$ insulin. Matching was performed on all the baseline characteristics listed in Table 1 using the Greedy Match macro (Mayo Clinic College of Medicine; http://www.mayo.edu/ research/documents/gmatchsas/doc-10027248.sas, last accessed April 2014).

SAS version 9.2 (SAS Institute, Cary, NC, USA) was used for the statistical analyses. A $p$ value $<0.05$ was considered statistically significant for all analyses.

Ethics The study was approved by the Danish Data Protection Agency (J.no. 2007-58-015I.suite no. 00916 GEH-2010-001). Retrospective register-based studies do not need ethical approval in Denmark.

\section{Results}

A total of 87,924 individuals initiated a combination of GLTs (Fig. 1). Among 77,145 patients without previous MI or stroke, 11,081 (14\%) used SU+insulin and 16,910 (22\%) used metformin+insulin. Their baseline characteristics are presented in Table 1. Since some patients used more than one combination of drugs during the study period, the number of patients using each combination adds up to more than the total number of patients included $(n=25,404)$.

Compared with $\mathrm{SU}+$ insulin, patients receiving metformin + insulin were younger, had less comorbidity, had a longer duration of monotherapy treatment and had more often used metformin prior to initiating insulin combination therapy. Among individual SU+insulin combinations, patients receiving glibenclamide + insulin and glipizide + insulin were older than average, whereas patients receiving repaglinide+insulin were younger and had less comorbidity. Patients receiving gliclazide + insulin more often used cardiovascular pharmacotherapy.

$\mathrm{SU}+$ insulin was used by $16 \%$ of patients receiving a combination therapy in the initial phase of the study, but its use declined throughout the study period (Fig. 2). In 2009, SU+ insulin was used by $2,857(5.1 \%)$ of patients using dual combination therapies. The median treatment duration for $\mathrm{SU}+$ insulin combination therapy was 92 days (interquartile range [IQR] 44-216 days), and 788 (7.0\%) patients died while receiving this therapy. Among patients using $\mathrm{SU}+$ insulin, $3,346(30 \%)$ had a limited time of use ( $<2$ months) before 
Table 1 Baseline variables

\begin{tabular}{|c|c|c|c|c|c|c|c|}
\hline \multirow[t]{2}{*}{ Variable } & \multicolumn{6}{|l|}{ Insulin +} & \multirow[t]{2}{*}{ All } \\
\hline & Glibenclamide & Gliclazide & Glimepiride & Glipizide & Tolbutamide & Metformin & \\
\hline$n$ & 1,483 & 1,081 & 7,275 & 1,028 & 459 & 16,910 & 25,404 \\
\hline Men, $n(\%)$ & $822(55.4)$ & $580(53.7)$ & $3,918(53.9)$ & $547(53.2)$ & $248(54.0)$ & $9,355(55.3)$ & $13,991(55.1)$ \\
\hline Age, mean $\pm \mathrm{SD}$ & $64.24 \pm 14.5$ & $63.03 \pm 14.5$ & $62.98 \pm 14.7$ & $63.66 \pm 15$ & $63.59 \pm 14.9$ & $57.38 \pm 13.1$ & $59.61 \pm 14.2$ \\
\hline $\begin{array}{l}\text { Treatment duration in years, } \\
\text { median (IQR) }\end{array}$ & $3.39(0.9-5.4)$ & $3.86(1.4-6.0)$ & $3.41(0.9-5.4)$ & $3.37(0.8-5.3)$ & $2.6(0.3-4.1)$ & $4.07(1.2-6.4)$ & $3.07(0.8-5.7)$ \\
\hline \multicolumn{8}{|l|}{ Comorbidities, $n(\%)$} \\
\hline Congestive heart failure & $139(9.4)$ & $76(7.0)$ & $704(9.7)$ & $85(8.3)$ & $40(8.7)$ & $814(4.8)$ & $1,627(6.4)$ \\
\hline Atrial fibrillation & $114(7.7)$ & $88(8.1)$ & $682(9.4)$ & $77(7.5)$ & $38(8.3)$ & $819(4.8)$ & $1,628(6.4)$ \\
\hline Renal disease & $45(3.0)$ & $26(2.4)$ & $278(3.8)$ & $31(3.0)$ & $17(3.7)$ & $200(1.2)$ & $534(2.1)$ \\
\hline Peripheral vascular disease & $36(2.4)$ & $44(4.1)$ & $193(2.7)$ & $31(3.0)$ & $15(3.3)$ & $266(1.6)$ & $512(2.0)$ \\
\hline COPD & $103(7.0)$ & $65(6.0)$ & $514(7.1)$ & $72(7.0)$ & $26(5.7)$ & $728(4.3)$ & $1,332(5.2)$ \\
\hline Peptic ulcer & $71(4.8)$ & $52(4.8)$ & $429(5.9)$ & $52(5.1)$ & $23(5.0)$ & $522(3.1)$ & $1,023(4.0)$ \\
\hline Cancer & $143(9.6)$ & $99(9.2)$ & $694(9.5)$ & $94(9.1)$ & $54(11.8)$ & $857(5.1)$ & $1,766(7.0)$ \\
\hline \multicolumn{8}{|l|}{ Charlson score, $n(\%)$} \\
\hline 0 & $1,055(71.1)$ & 777 (71.9) & $5,097(70.1)$ & $713(69.4)$ & $310(67.5)$ & $14,039(83.0)$ & $19,831(78.1)$ \\
\hline $1-2$ & $378(25.5)$ & $254(23.5)$ & $1,790(24.6)$ & $271(26.4)$ & $115(25.1)$ & $2,542(15.0)$ & $4,785(18.8)$ \\
\hline$\geq 3$ & $50(3.4)$ & $50(4.6)$ & $388(5.3)$ & $44(4.3)$ & $34(7.4)$ & $329(2.0)$ & $788(3.1)$ \\
\hline \multicolumn{8}{|l|}{ Concomitant therapy, $n(\%)$} \\
\hline Statin & $423(28.5)$ & $458(42.4)$ & $2,929(40.3)$ & $324(31.5)$ & $98(21.4)$ & $9,290(54.9)$ & $11,830(46.6)$ \\
\hline RASi & $598(40.3)$ & $538(49.8)$ & $3,461(47.6)$ & $439(42.7)$ & $159(34.6)$ & $9,522(56.3)$ & $12,946(51.0)$ \\
\hline Beta-blocker & $311(21.0)$ & $245(22.7)$ & $1,804(24.8)$ & $223(21.7)$ & $79(17.2)$ & $3,910(23.1)$ & $5,834(23.0)$ \\
\hline Aspirin & $466(31.4)$ & $392(36.3)$ & $2,516(34.6)$ & $319(31.0)$ & $130(28.3)$ & $6,203(36.7)$ & $8,812(34.7)$ \\
\hline Thiazides & $333(22.5)$ & $228(21.1)$ & $1,705(23.4)$ & $219(21.3)$ & $94(20.5)$ & $4,153(24.6)$ & $6,004(23.6)$ \\
\hline Calcium antagonist & $286(19.3)$ & $275(25.4)$ & $1,721(23.7)$ & $225(21.9)$ & $100(21.8)$ & $4,116(24.3)$ & $5,929(23.3)$ \\
\hline Other antihypertensive & $30(2)$ & $39(3.6)$ & $188(2.6)$ & $25(2.4)$ & $12(2.6)$ & $464(2.7)$ & $659(2.6)$ \\
\hline Nitrates & $140(9.4)$ & $104(9.6)$ & $644(8.9)$ & $101(9.8)$ & $34(7.4)$ & $1,079(6.4)$ & $1,813(7.1)$ \\
\hline Anticoagulation therapy & $91(6.1)$ & $84(7.8)$ & $684(9.4)$ & $61(5.9)$ & $26(5.7)$ & $1,027(6.1)$ & $1,783(7.0)$ \\
\hline Digoxin & $202(13.6)$ & $122(11.3)$ & 868 (11.9) & $116(11.3)$ & $57(12.4)$ & $987(5.8)$ & $2,113(8.3)$ \\
\hline Spironolactone & $146(9.8)$ & $100(9.3)$ & $816(11.2)$ & $99(9.6)$ & $45(9.8)$ & $1,038(6.1)$ & $1,991(7.8)$ \\
\hline \multicolumn{8}{|l|}{ Inclusion period, $n(\%)$} \\
\hline January 1997-March 2000 & 355 (23.9) & $69(6.4)$ & $508(7.0)$ & $228(22.2)$ & $143(31.2)$ & $456(2.7)$ & $1,696(6.7)$ \\
\hline April 2000-June 2003 & $423(28.5)$ & $196(18.1)$ & $1,628(22.4)$ & $284(27.6)$ & $153(33.3)$ & 2,295 (13.6) & 4,539 (17.9) \\
\hline July 2003-September 2006 & 409 (27.6) & $425(39.3)$ & $2,680(36.8)$ & $285(27.7)$ & $113(24.6)$ & $5,558(32.9)$ & $8,616(33.9)$ \\
\hline October 2006-December 2009 & $296(20.0)$ & $391(36.2)$ & $2,458(33.8)$ & $231(22.5)$ & $50(10.9)$ & $8,601(50.9)$ & $10,552(41.5)$ \\
\hline \multicolumn{8}{|l|}{ First GLT used, $n(\%)$} \\
\hline Insulin & $80(5.4)$ & $49(4.5)$ & $547(7.5)$ & $58(5.6)$ & $30(6.5)$ & $1,748(10.3)$ & $2,343(9.2)$ \\
\hline SU & $1,206(81.3)$ & $800(74.0)$ & $5,071(69.7)$ & $810(78.8)$ & $393(85.6)$ & $6,709(39.7)$ & $13,094(51.5)$ \\
\hline Metformin & $120(8.1)$ & $179(16.6)$ & $1,217(16.7)$ & $121(11.8)$ & $22(4.8)$ & $6,714(39.7)$ & $7,772(30.6)$ \\
\hline Combination of GLTs & $58(3.9)$ & $26(2.4)$ & $338(4.7)$ & $27(2.6)$ & $9(2.0)$ & $1,409(8.3)$ & $1,754(6.9)$ \\
\hline Other & $19(1.3)$ & $27(2.5)$ & $102(1.4)$ & $12(1.2)$ & $5(1.1)$ & $330(2.0)$ & $441(1.7)$ \\
\hline Any prior use of SU, $n(\%)$ & $1,384(93.3)$ & $1,024(94.7)$ & $6,571(90.3)$ & $960(93.4)$ & $425(92.6)$ & $10,954(64.8)$ & $18,509(72.9)$ \\
\hline Any prior use of metformin, $n(\%)$ & $617(41.6)$ & $569(52.6)$ & $3,491(48)$ & $448(43.6)$ & $109(23.8)$ & $13,572(80.3)$ & $17,011(67.0)$ \\
\hline Prior combination treatment, $n(\%)$ & $56(3.8)$ & $79(7.3)$ & $524(7.2)$ & $36(3.5)$ & $20(4.4)$ & $2,263(13.4)$ & \\
\hline Previous hypoglycaemia, $n(\%)$ & $20(1.4)$ & $24(2.2)$ & $120(1.7)$ & $14(1.4)$ & $3(0.7)$ & $163(1.0)$ & $267(1.1)$ \\
\hline
\end{tabular}

Data are $n(\%)$ or mean \pm SD or median (IQR)

COPD, Chronic obstructive pulmonary disease; RASi, renin-angiotensin system inhibitor 


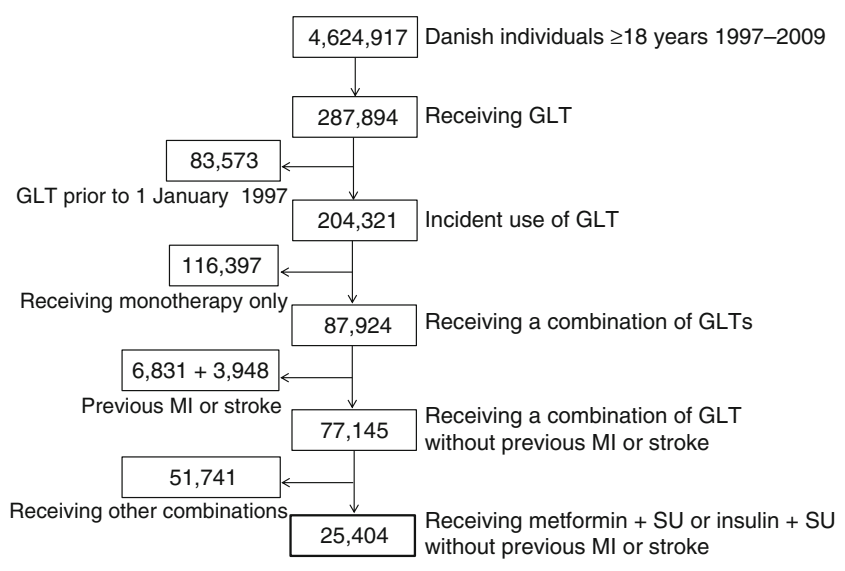

Fig. 1 Study flowchart

receiving another therapy, while after 1 year of treatment $1,899(18 \%)$ patients were still alive and were continuing to use SU+insulin. When stopping $\mathrm{SU}+$ insulin, most patients changed to insulin monotherapy (68\%), triple (or more) therapy $(13 \%)$ or SU monotherapy $(11 \%)$, while a few patients changed to metformin +insulin (3\%), metformin monotherapy $(<1 \%)$ or metformin $+\mathrm{SU}(<1 \%)$, or stopped claiming GLT $(5 \%)$.

Metformin+insulin was increasingly used throughout the study period. The median duration of treatment was 465 days (IQR 127-1,109 days), and 774 (4.6\%) patients died during the therapy. When stopping metformin +insulin, most patients changed to insulin monotherapy (55\%), triple (or more) therapy $(17 \%)$ or metformin monotherapy $(20 \%)$, while only a few patients changed to $\mathrm{SU}+$ insulin $(1 \%)$ or $\mathrm{SU}+$ metformin $(1 \%)$, or stopped claiming GLT $(5 \%)$.

Mortality and cardiovascular endpoints Crude incidence rates of mortality, cardiovascular death and the combined endpoint according to treatment are presented in Table 2 .

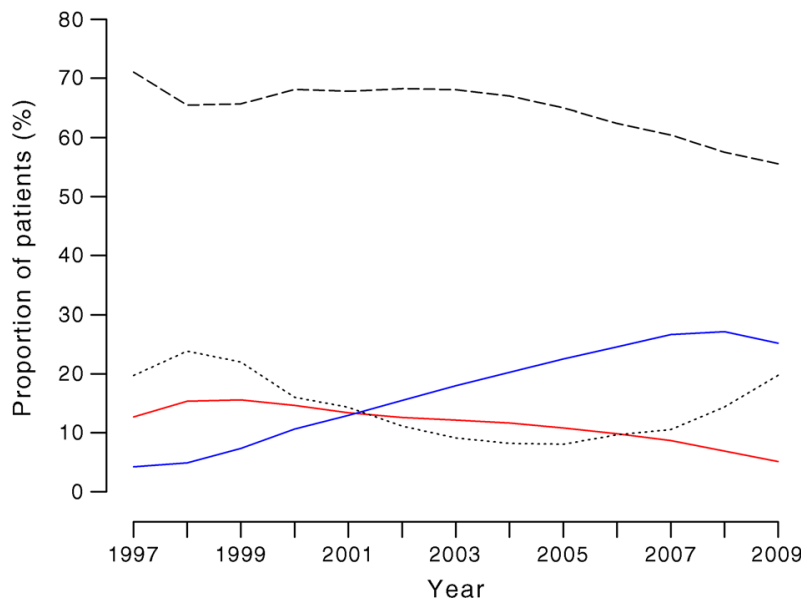

Fig. 2 Use of SU+insulin compared with other dual therapies according to year. Red line, $\mathrm{SU}+$ insulin; blue line, metformin + insulin; dashed line, metformin $+\mathrm{SU}$; dotted line, other dual therapy
$\mathrm{SU}+$ insulin was associated with mortality rates that were two to five times higher than combinations of metformin + insulin. Among individual combinations of SUs + insulin, glibenclamide, tolbutamide and glipizide were associated with the highest incidence rates for all endpoints, whereas the use of gliclazide was associated with lower rates.

RRs from multivariable analyses are presented in Fig. 3. All combinations of SU+insulin were associated with a significantly higher risk of mortality when compared with metformin + insulin. When pooling combinations of SUs + insulin, higher RRs were observed for all endpoints compared with metformin + insulin.

Using glimepiride + insulin as a reference, mortality was not statistically different (RR [95\% CI]) for combinations of insulin with glibenclamide (RR 1.13 [0.91, 1.41]), gliclazide (RR 0.84 [0.64, 1.09]), glipizide (RR 1.24 [0.96, 1.58]) and tolbutamide (RR 1.20 [0.83, 1.74]), and the differences in the risk of cardiovascular death and the combined endpoint were not statistically significant among individual SUs.

Sensitivity analyses The results were similar in the sensitivity analyses (see electronic supplementary material [ESM] Table 1): condition (a) using only the initial combination therapy (censoring patients if changing combination therapy), condition (b) excluding patients who had used insulin prior to combination therapy, condition (c) including only patients who had used solely SU prior to combination therapy, condition (d) using a continually updated Charlson score, and condition (e) including in the model a registered diagnosis of heart failure (ICD-10: I50.x, I42.x, I110 or J819), renal disease (ICD-8: 582-586, 588, T558-559, Z992; ICD-10: N03, N04, N19-N19, R34, I12.x-I13.x), chronic obstructive pulmonary disease (ICD 8: 490-492; ICD-10 J42, J44), peripheral vascular disease (ICD-8: 443; ICD-10: I70 and I74) and use of loop diuretics (ATC: C03C) instead of Charlson score.

For the propensity score model, the $\mathrm{C}$ statistic was 0.78 . The baseline characteristics were similar in the propensity score matched populations (ESM Table 2). Multivariable analysis on this matched population confirmed the results of the main analysis: the use of $\mathrm{SU}+$ insulin was associated with increased mortality (RR $1.70[1.48,1.95])$, cardiovascular death (RR $1.35[1.07,1.70])$ and the combined endpoint (RR $1.25[1.05,1.49])$.

Hypoglycaemia At baseline, 267 (1\%) patients had previously been hospitalised with hypoglycaemia (Table 1).

A total of $888(3.5 \%)$ patients were hospitalised with hypoglycaemia during the follow-up, the majority only once $(83 \%)$, but some two $(10.6 \%)$, three $(3.4 \%)$ or more $(3 \%)$ times.

Metformin +insulin was associated with lower incidence rates of hypoglycaemia compared with combinations of $\mathrm{SU}+$ insulin, while differences between SUs were less evident (Table 3). 
Table 2 Crude incidence rates per 1,000 person-years for mortality, cardiovascular death and a composite endpoint of stroke, MI and cardiovascular death

\begin{tabular}{|c|c|c|c|c|c|c|}
\hline \multirow[t]{2}{*}{ Variable } & \multicolumn{6}{|l|}{ Insulin +} \\
\hline & Glibenclamide & Gliclazide & Glimepiride & Glipizide & Tolbutamide & Metformin \\
\hline \multicolumn{7}{|l|}{ All-cause mortality } \\
\hline Events & 99 & 63 & 524 & 72 & 30 & 774 \\
\hline Person-years & 880 & 830 & 5,469 & 588 & 237 & 33,193 \\
\hline Crude incidence rates & $112(92-137)$ & $76(59-97)$ & $96(87-104)$ & $122(97-154)$ & $126(88-181)$ & $23(22-25)$ \\
\hline \multicolumn{7}{|l|}{ Cardiovascular death } \\
\hline Events & 36 & 19 & 188 & 20 & 9 & 325 \\
\hline Person-years & 880 & 830 & 5,469 & 588 & 237 & 33,193 \\
\hline Crude incidence rates & $40(29-56)$ & $23(15-36)$ & $34(30-40)$ & $33(22-52)$ & $38(20-73)$ & $9.8(8.8-10.9)$ \\
\hline \multicolumn{7}{|l|}{ Composite endpoint } \\
\hline Events & 52 & 36 & 265 & 37 & 13 & 650 \\
\hline Person-years & 850 & 809 & 5,321 & 578 & 235 & 32,451 \\
\hline Crude incidence rate & $61(47-80)$ & $46(32-62)$ & $49(44-56)$ & $64(46-88)$ & $55(32-95)$ & $20(18.6-21.6)$ \\
\hline
\end{tabular}

In the multivariable analysis, the RR of having a first hypoglycaemic event after baseline was significantly higher among users of SU+insulin compared with users of metformin +insulin (RR $2.06[1.62,2.61])$.

Hypoglycaemia prior to baseline was associated with an increased risk of all-cause mortality (RR 2.16 [1.94, 2.40]), cardiovascular death (RR $2.18[1.85,2.56])$ and the combined endpoint (RR 1.82 [1.56, 2.12]). However, including hypoglycaemia in the multivariable model did not markedly attenuate the risk associated with $\mathrm{SU}+$ insulin compared with metformin +insulin in terms of mortality (RR $1.82[1.64$, $2.02])$, cardiovascular death (RR $1.36[1.15,1.62])$ or the combined endpoint (RR $1.25[1.09,1.43])$.

\section{Discussion}

We investigated the use of insulin in combination with an SU or metformin and the associated mortality and cardiovascular risk in a nationwide setting and report four major findings.

First, a relatively large proportion of individuals using GLT received $\mathrm{SU}+$ insulin during the initial study period, although this declined substantially and was replaced with an increased use of metformin + insulin. Second, SU+insulin was associated with increased mortality compared with metformin+insulin when adjusting for potential confounding factors. Third, there were no significant differences between individual SUs in terms of mortality or cardiovascular endpoints when used in combination with insulin. Fourth, hypoglycaemia was associated with increased mortality and SU+insulin was associated with a higher risk of hypoglycaemia than metformin +insulin. However, the complexity of analyses in terms of accounting for the effects of past and present exposures to multiple risk factors and pharmacotherapies must be appreciated when interpreting the results.

To the best of our knowledge, there are no previous studies on the trajectories of prescription patterns and the use of different combinations of oral glucose-lowering drugs with insulin. The increasing use of metformin is consistent with the report in 1998 of its beneficial effect [18]. Furthermore, a study in 1999 [19] claimed significant benefits with respect to weight gain, change in $\mathrm{HbA}_{1 \mathrm{c}}$ and risk of hypoglycaemia with the use metformin +insulin compared with insulin alone or SU+insulin. These studies are likely to offer an explanation for the change in use of insulin combination treatments.

$\mathrm{SU}+$ insulin was associated with higher mortality than metformin+insulin. In a study by Lund et al [20], metformin+insulin, when compared with repaglinide+ insulin, was associated with a similar improvement in glucose control but less weight gain after 12 months [20]. However, to the best of our knowledge, there are no large studies on longterm outcomes with the use of SU+insulin compared with metformin +insulin. Our findings suggest that the use of metformin +insulin is associated with an improved prognosis compared with the use of SU+insulin. Although the reason for this is unknown, it may be speculated that a lower weight gain and a lower risk of hypoglycaemia with metformin+ insulin compared with SU+insulin may play a role. There has long been controversy surrounding the potential cardiovascular and mortality risk associated with the overall use of SUs [21], and studies comparing the risk from SUs with those from other GLTs have yielded conflicting results [11, 12, 22-24].

SUs promote insulin secretion by binding to SU receptors in pancreatic beta cells (SUR1) and inhibiting potassium- 
a

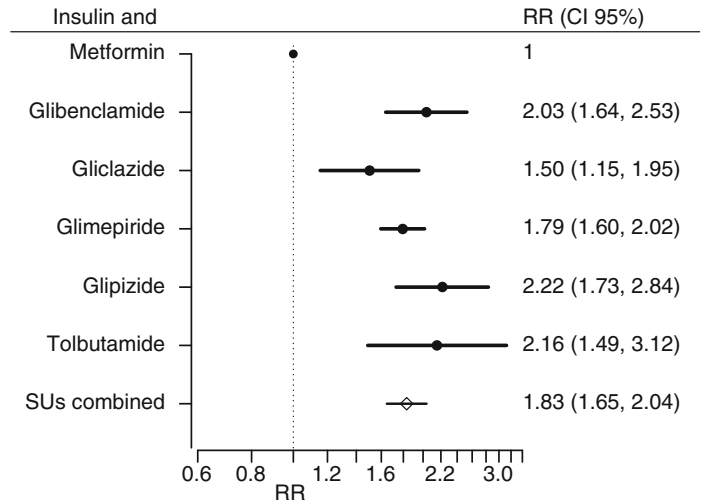

b

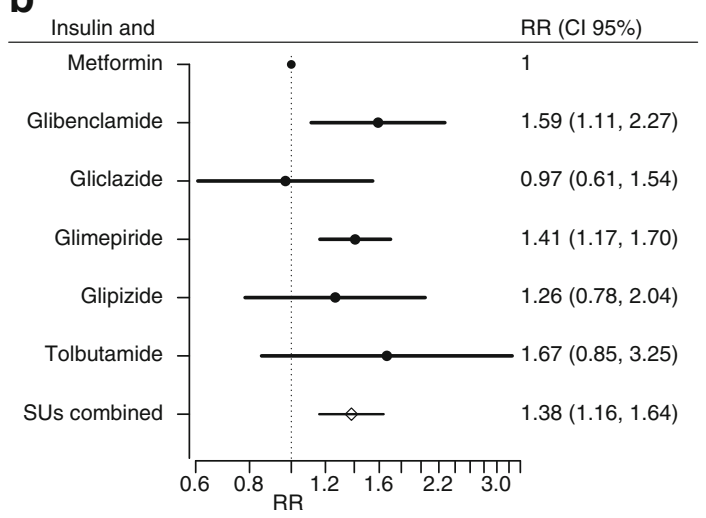

C

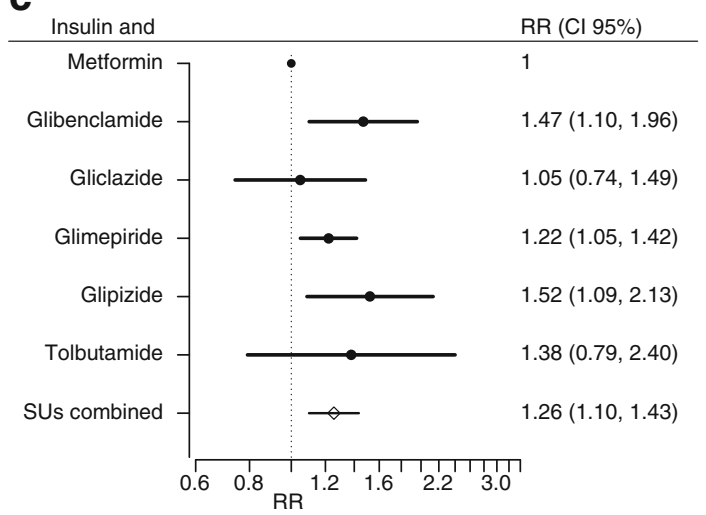

Fig. 3 RRs and 95\% CIs associated with the use of insulin in combination with SUs or metformin for (a) all-cause mortality, (b) cardiovascular death, and (c) the composite endpoint of acute MI, stroke and cardiovascular death sensitive adenosine triphosphate. Since SUs vary in their specificity and similar receptors can be found in the myocardium (SUR2A) and smooth muscle cells (SUR2B), it has been speculated that SU therapy could interfere with protective adaptations to prior ischaemia known as ischaemic preconditioning [25]. Glibenclamide in particular has been associated with an inhibition of ischaemic preconditioning [26, 27], in contrast to glimepiride [27] and gliclazide [28] (with a higher affinity for SUR1). In this study, gliclazide use was associated with the lowest incidence rates of adverse outcomes, consistent with the above speculations. Even though it cannot be excluded that our study lacked sufficient statistical power, the lower risk associated with gliclazide use was not significant in adjusted analyses.

In other studies on clinical outcome data, glibenclamide was associated with increased mortality when compared with glimepiride and gliclazide [10, 11], and it has been speculated that the increased mortality associated with intensive therapy in the Action to Control Cardiovascular Risk in Diabetes (ACCORD) study [15], a signal not observed in the Action in Diabetes and Vascular Disease: Preterax and Diamicron MR Controlled Evaluation (ADVANCE) study [13], might be underpinned by a differential use of SUs $[11,14]$. Hence, glimepiride and glibenclamide were frequently used in ACCORD, while gliclazide was used as part of the protocol in ADVANCE.

We observed no statistically significant differences between individual SUs in terms of mortality or cardiovascular risk when used in combination with insulin, consistent with previous reports of a lack of difference in risk between SUs $[29,30]$. Thus, if differences in mortality risk exist between SUs, combination with insulin seems to eliminate this.

Hypoglycaemia was more frequent with the use of $\mathrm{SU}+$ insulin, consistent with reports of a doubling in rates of hypoglycaemia among users of SU+insulin compared with metformin+insulin or insulin alone [19]. In other studies, $\mathrm{SU}+$ insulin was associated with only mild hypoglycaemic reactions [5, 31]. Nevertheless, it has been speculated that the use of SU+insulin causing hypoglycaemia could be one explanation for the increased mortality in the intensive therapy arm in ACCORD [9]. We found hypoglycaemia to be

Table 3 Crude incidence rates for hypoglycaemia

\begin{tabular}{lllllll}
\hline Variable & \multicolumn{2}{l}{ Insulin +} & & & \\
\cline { 5 - 7 } & Glibenclamide & Gliclazide & Glimepiride & Glipizide & Tolbutamide & Metformin \\
\hline Hypoglycaemia & & & & & \\
$\quad$ Events & 20 & 17 & 118 & 10 & 5 & 206 \\
Person-years & 880 & 830 & 5,469 & 588 & 237 & 33,193 \\
Crude incidence rate per 1,000 person-years & $23(16-37)$ & $20(12-34)$ & $21(18-26)$ & $17(9-31)$ & $21(9-51)$ & $6(5-7)$ \\
\hline
\end{tabular}


associated with increased mortality, consistent with previous reports of associations with cardiovascular death $[32,33]$ and all-cause mortality (HR 2.69; 95\% CI 1.97, 3.67) [33]. However, adjusting for hypoglycaemia in the multivariable model did not significantly attenuate the difference in mortality between $\mathrm{SU}+$ insulin and metformin+insulin. This might be due to low sensitivity of identifying the most severe hypoglycaemic incidences as a cause of death (i.e. death could be presumed to be of other causes), but it also indicates that other factors contributed to the increased mortality. In a longterm follow-up analysis of the ACCORD study, hypoglycaemia did not appear to account for the increased mortality in the intensive therapy arm [34], indicating that hypoglycaemia might be a marker of adverse outcome rather than a direct causal link [33]. Whether hypoglycaemia is more frequent with certain combinations of GLTs and might be linked to increased mortality warrants further study.

One of the strengths of this study is the use of nationwide registries with complete information on all dispensed glucoselowering treatments, avoiding selection bias. However, our study has limitations as information on BMI, BP, dyslipidaemia, $\mathrm{HbA}_{1 \mathrm{c}}$ and smoking was not available, and these factors could potentially act as confounders. Instead, we adjusted for concomitant cardiovascular pharmacotherapy, household income and comorbidity, including kidney disease and heart failure. However, given the considerable baseline differences between users of metformin+insulin and $\mathrm{SU}+$ insulin, the possibility of residual confounding cannot be excluded.

We observed differences in the use of concomitant therapy, including statins, between different groups of SU users and metformin. One explanation could be differences in the time of inclusion, as a higher proportion of users of glibenclamide and metformin was included in the first part of the study, whereas users of, for example, gliclazide were generally included later. An increased use of statin therapy among patients with type 2 diabetes around the millennium might explain the prescription differences. To account for these differences, we adjusted for calendar year of inclusion in all analyses.

A previous restrictive use of metformin in patients with heart failure and renal disease and a fear of lactate acidosis is likely to introduce confounding by indication. Furthermore, we cannot exclude the fact that the shorter treatment duration of SU+insulin might have occurred because patients were actually changing to insulin monotherapy. Nevertheless, sensitivity analyses and propensity score matched analyses yielded similar results. Potential differences between different types of insulin were not investigated in the present study. Finally, the diagnosis of hypoglycaemia has not been validated, and we did not have information on glucose levels. However, as serious hypoglycaemic episodes are likely to be correctly registered, and given their clinical importance, we find it relevant to report the data.
In conclusion, a combination of SU+insulin is being used less frequently, in accordance with guidelines. In adjusted analyses, SU+insulin was associated with increased mortality compared with metformin + insulin. There were no statistically significant differences in risk between individual SUs in combinations with insulin. Hypoglycaemia was more frequent with $\mathrm{SU}+$ insulin compared with metformin + insulin and was associated with an increased risk. Differences in baseline characteristics between treatment groups and the observational design should be considered when interpreting the results.

Funding This work was supported by a grant from the Interreg IVA program, a part of the European Union. CA was supported by an independent research grant from the Danish Agency for Science, Technology and Innovation (grant number FSS - 11-120873). GG is supported by an independent research scholarship from the Novo Nordisk Foundation.

Duality of interest $\mathrm{AV}$ owns stocks and has received honoraria for lectures and for consultancy services from Novo Nordisk. GG owns stocks in Novo Nordisk. All other authors declare that there is no duality of interest associated with their contribution to this manuscript.

Contribution statement UMM made the primary contributions, researched the data and wrote the manuscript. CA, ELF and GG researched the data. All authors made substantial contributions to the interpretation of the data, revised the manuscript for intellectual content and approved the final version of the manuscript. UMM is responsible for the integrity of the work as a whole.

\section{References}

1. Turner RC, Cull CA, Frighi V, Holman RR (1999) Glycemic control with diet, sulfonylurea, metformin, or insulin in patients with type 2 diabetes mellitus: progressive requirement for multiple therapies (UKPDS 49). UK Prospective Diabetes Study (UKPDS) Group. JAMA 281:2005-2012

2. Nathan DM, Buse JB, Davidson MB et al (2009) Medical management of hyperglycaemia in type 2 diabetes: a consensus algorithm for the initiation and adjustment of therapy: a consensus statement of the American Diabetes Association and the European Association for the Study of Diabetes. Diabetologia 52:17-30

3. Inzucchi SE, Bergenstal RM, Buse JB et al (2012) Management of hyperglycaemia in type 2 diabetes: a patient-centered approach. Position statement of the American Diabetes Association (ADA) and the European Association for the Study of Diabetes (EASD). Diabetologia 55:1577-1596

4. Nathan DM (2002) Clinical practice. Initial management of glycemia in type 2 diabetes mellitus. N Engl J Med 347:1342-1349

5. Wright A, Burden AC, Paisey RB, Cull CA, Holman RR (2002) Sulfonylurea inadequacy: efficacy of addition of insulin over 6 years in patients with type 2 diabetes in the U.K. Prospective Diabetes Study (UKPDS 57). Diabetes Care 25:330-336

6. Pugh JA, Wagner ML, Sawyer J, Ramirez G, Tuley M, Friedberg SJ (1992) Is combination sulfonylurea and insulin therapy useful in NIDDM patients? A metaanalysis. Diabetes Care 15:953-959

7. Gaede P, Vedel P, Larsen N, Jensen GV, Parving HH, Pedersen O (2003) Multifactorial intervention and cardiovascular disease in patients with type 2 diabetes. N Engl J Med 348:383-393 
8. Lebovitz HE, Pasmantier R (1990) Combination insulin-sulfonylurea therapy. Diabetes Care 13:667-675

9. Dluhy RG, McMahon GT (2008) Intensive glycemic control in the ACCORD and ADVANCE trials. N Engl J Med 358:2630-2633

10. Khalangot M, Tronko M, Kravchenko V, Kovtun V (2009) Glibenclamide-related excess in total and cardiovascular mortality risks: data from large Ukrainian observational cohort study. Diabetes Res Clin Pract 86:247-253

11. Schramm TK, Gislason GH, Vaag A et al (2011) Mortality and cardiovascular risk associated with different insulin secretagogues compared with metformin in type 2 diabetes, with or without a previous myocardial infarction: a nationwide study. Eur Heart J 32:1900-1908

12. Gangii AS, Cukierman T, Gerstein HC, Goldsmith CH, Clase CM (2007) A systematic review and meta-analysis of hypoglycemia and cardiovascular events: a comparison of glyburide with other secretagogues and with insulin. Diabetes Care 30:389-394

13. Patel A, MacMahon S, Chalmers J et al (2008) Intensive blood glucose control and vascular outcomes in patients with type 2 diabetes. N Engl J Med 358:2560-2572

14. Gore MO, McGuire DK (2011) Resolving drug effects from class effects among drugs for type 2 diabetes mellitus: more support for cardiovascular outcome assessments. Eur Heart J 32:1832-1834

15. Gerstein HC, Miller ME, Byington RP et al (2008) Effects of intensive glucose lowering in type 2 diabetes. N Engl J Med 358:2545-2559

16. Olsen AM, Fosbol EL, Lindhardsen J et al (2013) Cause-specific cardiovascular risk associated with nonsteroidal anti-inflammatory drugs among myocardial infarction patients - a nationwide study. PLoS One 8:e54309

17. Thygesen SK, Christiansen CF, Christensen S, Lash TL, Sorensen HT (2011) The predictive value of ICD-10 diagnostic coding used to assess Charlson comorbidity index conditions in the population-based Danish National Registry of Patients. BMC Med Res Methodol 11:83

18. UK Prospective Diabetes Study (UKPDS) Group (1998) Intensive blood-glucose control with sulphonylureas or insulin compared with conventional treatment and risk of complications in patients with type 2 diabetes (UKPDS 33). Lancet 352:837-853

19. Yki-Jarvinen H, Ryysy L, Nikkila K, Tulokas T, Vanamo R, Heikkila M (1999) Comparison of bedtime insulin regimens in patients with type 2 diabetes mellitus. A randomized, controlled trial. Ann Intern Med 130: 389-396

20. Lund SS, Tarnow L, Frandsen M et al (2009) Combining insulin with metformin or an insulin secretagogue in non-obese patients with type 2 diabetes: 12 month, randomised, double blind trial. BMJ 339:b4324

21. Salsburg DS (1971) The UGDP study. JAMA 218:1704-1705
22. Kahler KH, Rajan M, Rhoads GG et al (2007) Impact of oral antihyperglycemic therapy on all-cause mortality among patients with diabetes in the Veterans Health Administration. Diabetes Care 30:1689-1693

23. Tzoulaki I, Molokhia M, Curcin V et al (2009) Risk of cardiovascular disease and all cause mortality among patients with type 2 diabetes prescribed oral antidiabetes drugs: retrospective cohort study using UK general practice research database. BMJ 339:b4731

24. Roumie CL, Hung AM, Greevy RA et al (2012) Comparative effectiveness of sulfonylurea and metformin monotherapy on cardiovascular events in type 2 diabetes mellitus: a cohort study. Ann Intern Med 157:601-610

25. Gribble FM, Reimann F (2003) Differential selectivity of insulin secretagogues: mechanisms, clinical implications, and drug interactions. J Diabetes Complicat 17:11-15

26. Tomai F, Crea F, Gaspardone A et al (1994) Ischemic preconditioning during coronary angioplasty is prevented by glibenclamide, a selective ATP-sensitive K+ channel blocker. Circulation 90:700-705

27. Klepzig H, Kober G, Matter C et al (1999) Sulfonylureas and ischaemic preconditioning; a double-blind, placebo-controlled evaluation of glimepiride and glibenclamide. Eur Heart J 20:439-446

28. Maddock HL, Siedlecka SM, Yellon DM (2004) Myocardial protection from either ischaemic preconditioning or nicorandil is not blocked by gliclazide. Cardiovasc Drugs Ther 18:113-119

29. Evans JM, Ogston SA, Reimann F, Gribble FM, Morris AD, Pearson ER (2008) No differences in mortality between users of pancreatic-specific and non-pancreatic-specific sulphonylureas: a cohort analysis. Diabetes Obes Metab 10:350-352

30. Andersson C, Gislason GH, Jorgensen CH et al (2011) Comparable long-term mortality risk associated with individual sulfonylureas in diabetes patients with heart failure. Diabetes Res Clin Pract 94:119 125

31. Groop LC, Groop PH, Stenman S (1990) Combined insulinsulfonylurea therapy in treatment of NIDDM. Diabetes Care 13(Suppl 3):47-52

32. Goto A, Arah OA, Goto M, Terauchi Y, Noda M (2013) Severe hypoglycaemia and cardiovascular disease: systematic review and meta-analysis with bias analysis. BMJ 347:f4533

33. Zoungas S, Patel A, Chalmers J et al (2010) Severe hypoglycemia and risks of vascular events and death. N Engl J Med 363:1410-1418

34. Bonds DE, Miller ME, Bergenstal RM et al (2010) The association between symptomatic, severe hypoglycaemia and mortality in type 2 diabetes: retrospective epidemiological analysis of the ACCORD study. BMJ 340:b4909 